\title{
NILPOTENT SPACES OF SECTIONS
}

\author{
JESPER MICHAEL MøLLER
}

\begin{abstract}
The space of sections of a fibration is nilpotent provided the base is finite $C W$-complex and the fiber is nilpotent. Moreover, localization commutes with the formation of section spaces.
\end{abstract}

1. Introduction. In their famous book on localization theory and later in the proceedings of the Vancouver 1977 conference, Hilton et al. showed that (any component of) the space of maps of a finite $C W$-complex into a nilpotent space is itself nilpotent [5, II. 2.6; 6, Theorem A]. The main purpose of this paper is to state and prove a twisted and relative version of their result.

To be more precise, let now, and throughout the paper, $A \stackrel{i}{\rightarrow} X$ be a cofibration, $p: Y \rightarrow B$ a fibration with fiber $F, u: X \rightarrow Y$ a continuous map, and let

$$
F_{u}(X, A ; Y, B):=\{v: X \rightarrow Y \mid v i=u i, p v=p u\}
$$

be the space of all lifts of $p u: X \rightarrow B$ which agree with $u$ on $A$. In this set-up, $F_{u}(X, A ; Y, B)$ is a nilpotent space provided $F$ is nilpotent and $(X, A)$ is a finite relative $C W$-complex (Theorem 4.1).

The proof of this assertion proceeds essentially as in the case considered by Hilton et al. of ordinary mapping spaces. A key move, though, is to establish a usable generalization of the principally refined Postnikov systems of nilpotent spaces. This is done in $\S 3$ and could be of some independent interest.

The content of the remaining sections is as follows. $\$ 2$ contains the basic definitions, a few preliminary lemmas, and nilpotency of the function space is proved in a particularly easy case corresponding to the easy construction of localization functors in the simply connected category. Nilpotency in the general case is proved in $\$ 4$ by means of the refined Postnikov towers of $\S 3$. This corresponds to the standard way of constructing localization functors in the nilpotent category. Finally, in $\S 5$, we show that fiberwise localization induces localization of the function space.

The following conventions are in force throughout the paper: $X$, the base $B$, and the fiber $F$ are 0-connected spaces; a space is said to be nilpotent if all its

Received by the editors November $11,1986$.

1980 Mathematics Subject Classification (1985 Revision). Primary 55P60; Secondary 55S45.

$K e y$ words and phrases. Section space, twisted Eilenberg-Mac Lane space, $K$-principal fibration. Postnikov decomposition, equivariant function space, localization. 
components are nilpotent; $G$ denotes an arbitrary group and $M$ a $G$-module; the function space $F_{u}(X, A ; Y, B)$ is equipped with the compactly generated topology associated to the compact-open topology.

2. The case of a simple fiber. In this section we recall some basic facts, introduce the notation, and prove the main result in a particularly easy case.

The $G$-action on $M$ can be realized geometrically as a based, cellular action on the Eilenberg-Mac Lane space $K(M, n+1), n>0$. Let $E G$ be a free, contractible $G$-space and put

$$
L(M, n+1):=E G \times_{G} K(M, n+1) .
$$

The obvious maps

$$
L(M, n+1) \underset{\check{h}}{\stackrel{\hat{h}}{\rightleftarrows}} K(G, 1)
$$

make $L(M, n+1)$ into an object in the category $\mathscr{T} K(G, 1)$ of spaces over and under $K(G, 1)[\mathbf{1 0}]$.

Let now $N$ be another $G$-module and $\operatorname{Hom}_{G}(M, N)$ the set of $G$-module homomorphisms of $M$ into $N$. Also, let $\langle L(M, n+1), L(N, n+1)\rangle_{K(G, 1)}$ denote the set of $\mathscr{T} K(G, 1)$-homotopy classes of maps of $L(M, n+1)$ into $L(N, n+1)$.

LEMMA 2.1. There is a bijective correspondence

$$
\operatorname{Hom}_{G}(M, N) \leftrightarrow\langle L(M, n+1), L(N, n+1)\rangle_{K(G, 1)} .
$$

Proof. By the universal nature of $\hat{k}: L(N, n+1) \rightarrow K(G, 1)$,

$$
\langle L(M, n+1), L(N, n+1)\rangle_{K(G, 1)}=H^{n+1}(L(M, n+1), K(G, 1) ; N) .
$$

The Serre spectral sequence for $\hat{k}: L(M, n+1) \rightarrow K(G, 1)$ with local coefficients $N$ shows [10, (5) p. 4] that $H^{n+1}(L(M, n+1), K(G, 1) ; N)=\operatorname{Hom}_{G}(M, N)$.

Recall that we have a path fibration

$$
\bar{P} L(M, n+1) \rightarrow L(M, n+1)
$$

in $\mathscr{T} K(G, 1)$. The path space is defined $[\mathbf{1 0}]$ as

$$
\bar{P} L(M, n+1):=\{\sigma: I \rightarrow L(M, n+1) \mid \hat{k} \sigma(I)=\hat{k} \sigma(0), \sigma(0)=\breve{k} \hat{k} \sigma(0)\}
$$

and the projection onto $L(M, n+1)$ is evaluation at $1 \in I=[0,1]$ as usual.

Let now $v: X \rightarrow \bar{P} L(M, n+1)$ be any map into this path space. Using the fact that $\hat{k}: \bar{P} L(M, n+1) \rightarrow K(G, 1)$ is a homotopy equivalence, one may prove

Lemma 2.2. $F_{v}(X, A ; \bar{P} L(M, n+1), K(G, 1))$ is contractible.

Let $Z \stackrel{f}{\rightarrow} K$ be a space over some other space $K$ and let $L \rightleftarrows K$ be an object of $\mathscr{T} K$.

Definition 2.3. A $K$-principal fibration over $Z$ is any fibration obtained as the pullback of the path fibration $\bar{P} L \rightarrow L$ in $\mathscr{T} K$ along a map $k: Z \rightarrow L$ over $K$.

Suppose for instance that the base space $B$ is a space over $K, B \stackrel{f}{\rightarrow} K$, so that any space over $B$ also becomes a space over $K$. Suppose furthermore that we have a 
commutative diagram of the form

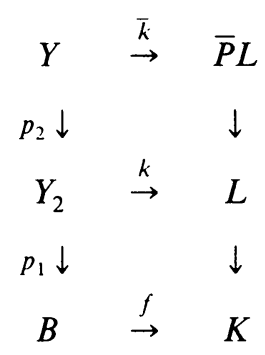

where $p=p_{1} p_{2}$ and $p_{2}: Y \rightarrow Y_{2}$ is the $K$-principal fibration defined by $k$. Put $u_{2}=p_{2} u$ and $u_{1}=p_{1} u_{2}$. In this situation we have the simple but important

Lemma 2.4. If $F_{u_{2}}\left(X, A ; Y_{2}, B\right)$ is nilpotent, so is $F_{u}(X, A ; Y, B)$.

Proof. The pullback diagram of the $K$-principal fibration $p_{2}$ induces another pullback diagram

$$
\begin{array}{ccc}
F_{u}(X, A ; Y, B) & \rightarrow & F_{\bar{k} u}(X, A ; \bar{P} L, K) \\
\downarrow & & \downarrow \\
F_{u}\left(X, A ; Y_{2}, B\right) & \rightarrow & F_{k u}(X, A ; L, K)
\end{array}
$$

of function spaces. Here, $F_{\bar{k} u}(X, A ; \bar{P} L, K)$ is contractible by Lemma 2.2, so the assertion follows from [5, II.2.2]. The requirement of [5, II.2.2] that all spaces be connected can be met, as in the proof of [6, Theorem A], by introducing suitable covering spaces of $F_{k u}(X, A ; L, K)$.

The material introduced at this point suffices to verify the nilpotency statement in a special case. Recall that $F$ is simple if $\pi_{1}(F)$ acts trivially on $\pi_{*}(F)$, that $F$ is finitely anticonnected if $\pi_{j}(F)=0$ for $j$ sufficiently large, and that $(X, A)$ is finitely coconnected if $H^{j}(X, A ; M)=0$ for any local coefficient system $M$ for $j$ sufficiently large.

Proposition 2.5. Suppose that the fiber $F$ is simple and that either $(X, A)$ is finitely coconnected or $F$ is finitely anticonnected. Then $F_{u}(X, A ; Y, B)$ is nilpotent.

Proof. Make $B$ into a space over $K(G, 1)$, where $G:=\pi_{1}(B)$, by choosing a map $B \rightarrow K(G, 1)$ which is the identity on $\pi_{1}$.

Since $F$ is simple, $G$ acts on $\pi_{*}(F)$ and we may erect Postnikov towers [10]

$$
\begin{aligned}
& L\left(\pi_{i} F, i+1\right)
\end{aligned}
$$

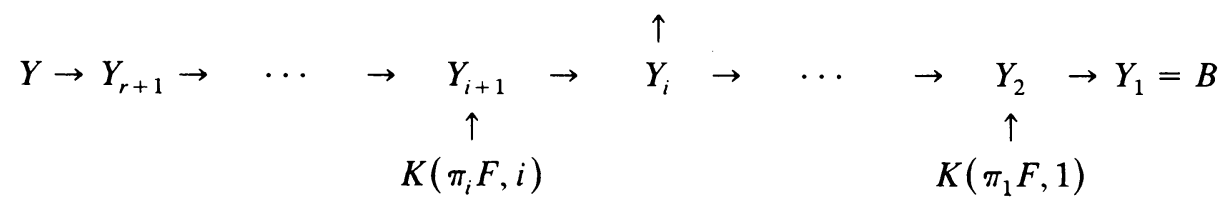

where $Y \rightarrow Y_{r+1}$ is $(r+1)$-connected and each stage $Y_{i+1} \rightarrow Y_{i}, 1 \leqslant i \leqslant r$, is a $K(G, 1)$-principal fibration. $F_{u}\left(X, A ; Y_{2}, B\right)$ is nilpotent, even simple, by [12], so repeated applications of Lemma 2.4 show that $F_{u}\left(X, A ; Y_{r+1}, B\right)$ is nilpotent. But 
the stated finiteness assumptions assure that this space converges to $F_{u}(X, A ; Y, B)$ as $r$ goes to infinity. Hence the latter space is nilpotent.

It is not true in general that $F_{u}(X, A ; Y, B)$ is simple even though $F$ is simple: The fundamental group of the space of degree 0 maps $S^{1} \times S^{1} \rightarrow S^{2}$ is not abelian $[2,8]$.

3. Refinements of Postnikov towers. Nilpotent spaces have the important property that their Postnikov systems admit principal refinements. In this section we shall relativize this concept.

First, we consider fibrations with acyclic fibers.

Let $N$ be a nilpotent group. The lower central series of $N$,

$$
\Gamma^{1}(N) \triangleright \Gamma^{2}(N) \triangleright \cdots \triangleright \Gamma^{i}(N) \triangleright \Gamma^{i+1}(N) \triangleright \cdots,
$$

defined inductively by $\Gamma^{1}(N)=N, \Gamma^{i+1}(N)=\left[N, \Gamma^{i}(N)\right], i \geqslant 1$, is finite, in the sense that $\Gamma^{c+1}(N)=\{1\}$ for some $c$, by the very definition of nilpotency. Any automorphism of $N$ restricts to an automorphism of $\Gamma^{i}(N)$ for all $i \geqslant 1$. In other words, $\operatorname{Aut}(N)$ acts on $\Gamma^{\mathrm{i}}(N)$ and hence also on $\Gamma^{i}(N) / \Gamma^{i+1}(N)$ for all $i \geqslant 1$. Moreover, inner automorphisms act trivially on $\Gamma^{i}(N) / \Gamma^{i+1}(N)$ and hence $\operatorname{Out}(N):=\operatorname{Aut}(N) / N$ acts on these subquotients. Similarly, $\operatorname{Aut}(N) / \Gamma^{i}(N)$ acts on $\Gamma^{i}(N) / \Gamma^{i+1}(N)$ and even on $H_{1}\left(\Gamma^{i}(N)\right)=\Gamma^{i}(N) /\left[\Gamma^{i}(N), \Gamma^{i}(N)\right]$.

Now assume that $K(N, 1) \rightarrow Y \rightarrow B$ is a fibration with an acyclic, nilpotent fiber. The associated semiaction [4, p. 142] of $\pi_{1}(B)$ on $N$ is a homomorphism $\psi$ : $\pi_{1}(B) \rightarrow \operatorname{Out}(N)$. $B$, and hence also $Y$, becomes a space over $K(\operatorname{Out}(N), 1)$ if we realize $\psi$ geometrically as a map of $B$ into $K(\operatorname{Out}(N), 1)$.

Lemma 3.1. Suppose that the fibration $p: Y \rightarrow B$ has an acyclic, nilpotent space $K(N, 1)$ as fiber. Then there is a factorization

$$
\begin{gathered}
L\left(\Gamma^{i} / \Gamma^{i+1}, 2\right) \\
\uparrow=Y_{c+1} \rightarrow Y_{c} \rightarrow \cdots \rightarrow Y_{i+1}^{\uparrow} \rightarrow Y_{i} \rightarrow \cdots \rightarrow Y_{1}=B \\
K\left(\Gamma^{i} / \Gamma^{i+1}, 1\right)
\end{gathered}
$$

of $p: Y \rightarrow B$ into a finite string of $K(\operatorname{Out}(N), 1)$-principal fibrations.

Proof. W.l.o.g., $p$ is the universal example [4, Theorem 2.1] of such a fibration. Then $Y=K(\operatorname{Aut}(N), 1)$ and $\pi_{1}(B)=\operatorname{Out}(N)$. In the Serre spectral sequence for $p$ with local coefficients $\operatorname{Out}(N) \rightarrow \operatorname{Aut}\left(\Gamma^{1}(N) / \Gamma^{2}(N)\right)$ one has, as in the proof of [10, Theorem 4.1], that

$$
\begin{aligned}
E_{2}^{01} & =\operatorname{Hom}_{\operatorname{Aut}(N) / \Gamma^{1}(N)}\left(H_{1} \Gamma^{1}(N), \Gamma^{1}(N) / \Gamma^{2}(N)\right) \\
& =\operatorname{Hom}_{\operatorname{Aut}(N)}\left(\Gamma^{1}(N), \Gamma^{1}(N) / \Gamma^{2}(N)\right) .
\end{aligned}
$$

Hence the projection map $\Gamma^{1}(N) \rightarrow \Gamma^{1}(N) / \Gamma^{2}(N)$, which is $\operatorname{Aut}(N)$-equivariant, is geometrically realizable $\left[\mathbf{1 0}\right.$, p. 5 and Theorem 3.1] by a map $Y_{1} \rightarrow$ $L\left(\Gamma^{1}(N) / \Gamma^{2}(N), 2\right)$ over $K(\operatorname{Out}(N), 1)$. By pullback of the path fibration we get a 
factorization

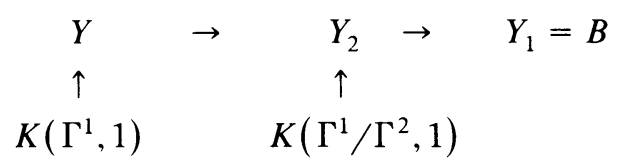

of $Y \rightarrow B$ such that $Y_{2} \rightarrow Y_{1}$ is a $K(\operatorname{Out}(N), 1)$-principal fibration and the restriction of $Y \rightarrow Y_{2}$ to the fibre induces the projection $\Gamma^{1}(N) \rightarrow \Gamma^{1}(N) / \Gamma^{2}(N)$ on $\pi_{1}$. This implies that the homotopy fiber of $Y \rightarrow Y_{2}$ is $K\left(\Gamma^{2}(N), 1\right)$ and $\pi_{1}\left(Y_{2}\right)=$ $\operatorname{Aut}(N) / \Gamma^{2}(N)$.

Repeat the argument with $Y_{1}$ replaced by $Y_{2}$, etc. The process eventually stops, because the homotopy fiber, $K\left(\Gamma^{c+1}(N), 1\right)$, of $Y \rightarrow Y_{c+1}$ is contractible if $\Gamma^{c+1}(N)=1$.

Next, we consider the abelian case. Suppose that the group $G$ fits into an exact sequence of groups of the form

$$
N \stackrel{\kappa}{\rightarrow} G \stackrel{\lambda}{\rightarrow} Q \rightarrow 1 .
$$

Then $N$ acts on $M$ through $\kappa$. Define the lower central $N$-series of $M$,

$$
\Gamma_{N}^{1}(M) \supset \Gamma_{N}^{2}(M) \supset \cdots \supset \Gamma_{N}^{i}(M) \supset \Gamma_{N}^{i+1}(M) \supset \cdots,
$$

by setting $\Gamma_{N}^{1}(M)=M$ and letting $\Gamma_{N}^{i+1}(M)$ be the subgroup generated by $\{n m-$ $\left.m \mid n \in N, m \in \Gamma_{N}^{i}(M)\right\}$. $N$ acts nilpotently on $M$ if $\Gamma_{N}^{c+1}(M)=0$ for some $c$.

LEMMA 3.2. $\Gamma_{N}^{i}(M)$ is a G-submodule for all $i \geqslant 1$.

Proof. For all $n \in N, m \in \Gamma_{N}^{i}(M)$, and $g \in G$,

$$
g(n m-m)=\left(g n g^{-1}\right) g m-g m \in \Gamma_{N}^{i+1}(M),
$$

for $g^{n} g^{-1} \in \kappa(N)$ and, arguing by induction, we may assume that $g m \in \Gamma_{N}^{i}(M)$.

Thus all the subquotients $\Gamma_{N}^{i}(M) / \Gamma_{N}^{i+1}(M)$ are $G$-modules and even $Q$-modules since $N$ acts trivially here.

Assume now that $K(M, n) \rightarrow Y \rightarrow B$ is a $K(G, 1)$-principal fibration, $n \geqslant 1$.

Lemma 3.3. Suppose that $N$ acts nilpotently on $M$. Then there is a factorization

$$
\begin{gathered}
L\left(\Gamma^{i} / \Gamma^{i+1}, n+1\right) \\
\uparrow=Y_{c+1} \rightarrow Y_{c} \rightarrow \quad \cdots \quad \rightarrow \quad Y_{i+1} \rightarrow Y_{i} \rightarrow \quad \cdots \quad \rightarrow Y_{1}=B \\
\\
K\left(\Gamma^{i} / \Gamma^{i+1}, n\right)
\end{gathered}
$$

of $p: Y \rightarrow B$ into a finite string of $K(Q, 1)$-principal fibrations.

Proof. W.l.o.g., $B=L(M, n+1)=E G \times{ }_{G} K(M, n+1), \quad Y=\bar{P} L(M, n+1)$, and $p: Y \rightarrow B$ is the path fibration in $\mathscr{T} K(G, 1)$.

Since the Milnor construction [7] of universal numerable, principal bundles is functorial, there exists a map $E \lambda: E G \rightarrow E Q$ over $\lambda: K(G, 1) \rightarrow K(Q, 1)$ such that $E \lambda(e g)=E \lambda(e) \lambda(g), e \in E G, g \in G$. By Lemma 2.1, there also exists a $G$-equivariant, based map

$$
\rho: K\left(\Gamma_{N}^{i}(M), n+1\right) \rightarrow K\left(\Gamma_{N}^{i}(M) / \Gamma_{N}^{i+1}(M), n+1\right)
$$


corresponding to the projection $\Gamma_{N}^{i}(M) \rightarrow \Gamma_{N}^{i}(M) / \Gamma_{N}^{i+1}(M)$ of $G$-modules. Considering the target as a $Q$-space, this means that $\rho(g x)=\lambda(g) \rho(x)$ for all $g \in G$ and $x \in K\left(\Gamma_{N}^{i}(M), n+1\right) . E \lambda \times \rho$ induces, by passing to orbit spaces, a map

$$
\begin{array}{ccc}
L\left(\Gamma_{N}^{i}(M), n+1\right) & \rightarrow & L\left(\Gamma_{N}^{i}(M) / \Gamma_{N}^{i+1}(M), n+1\right) \\
\downarrow \uparrow & & \downarrow \uparrow \\
K(G, 1) & \stackrel{\lambda}{\rightarrow} & K(Q, 1)
\end{array}
$$

over and under $\lambda$. Since this map lifts to the path spaces, we obtain, by forming pullback, a factorization

$$
\begin{array}{ccc}
\bar{P} L\left(\Gamma_{N}^{i}(M), n+1\right) & \rightarrow & Y_{i+1} \\
\uparrow & \uparrow & \uparrow \\
K\left(\Gamma^{i}, n\right) & K\left(\Gamma^{i} / \Gamma^{i+1}, n\right)
\end{array}
$$

of the path fibration over $L=L\left(\Gamma_{N}^{i}(M), n+1\right)$. Here, $Y_{i+1} \rightarrow L$ is a $K(Q, 1)$ principal fibration and the homotopy fiber of $\bar{P} L \rightarrow Y_{i+1}$ is $K\left(\Gamma_{N}^{i+1}(M), n\right)$. This implies that $Y_{i+1}=L\left(\Gamma_{N}^{i+1}(M), n+1\right)$ and $\bar{P} Y_{i+1}=\bar{P} L$.

If $N$ acts nilpotently on $M$, then this process eventually stops, for the homotopy fiber $K\left(\Gamma_{N}^{c+1}(M), n\right)$ of $\bar{P} L\left(\Gamma_{N}^{c}(M), n+1\right) \rightarrow Y_{c+1}$ is contractible if $\Gamma_{N}^{c+1}(M)=0$.

A combination of Lemma 3.1, Lemma 3.3, [4], and [10] yields

THEOREM 3.4. Let $p: Y \rightarrow B$ be a fibration with nilpotent fiber $F$. For $r \geqslant 1$ there exist factorizations

$$
Y \rightarrow Y_{s+1} \rightarrow \cdots \rightarrow Y_{i+1} \rightarrow Y_{i} \rightarrow \cdots \rightarrow Y_{2} \rightarrow Y_{1}=B
$$

of $p: Y \rightarrow B$ such that $Y \rightarrow Y_{s+1}$ is $(r+1)$-connected and each stage $Y_{i+1} \rightarrow Y_{i}$, $1 \leqslant i \leqslant s$, is a K-principal fibration where either $K=K\left(\right.$ Out $\left.\pi_{1}(F), 1\right)$ or $K=$ $K\left(\pi_{1}(B), 1\right)$.

Proof. There exist Postnikov decompositions of the form $[4,10]$ :

$$
\begin{aligned}
& Y \rightarrow Y_{r+1} \rightarrow \cdots \rightarrow Y_{i+1} \rightarrow Y_{i} \rightarrow \cdots \rightarrow Y_{2} \rightarrow Y_{1}=B \\
& \uparrow \\
& K\left(\pi_{i}(F), i\right) \quad K\left(\pi_{1}(F), 1\right)
\end{aligned}
$$

The first stage, $Y_{2} \rightarrow Y_{1}$, is a fibration of the type discussed in Lemma 3.1. The next stages, $Y_{i+1} \rightarrow Y_{i}, i \geqslant 2$, are $K\left(\pi_{1}(Y), 1\right)$-principal fibrations corresponding to the action of $\pi_{1}(Y)$ on $\pi_{i}(F)=\pi_{i+1}(B, Y)$. But $\pi_{1}(Y)$ fits into the exact sequence

$$
\pi_{1}(F) \rightarrow \pi_{1}(Y) \rightarrow \pi_{1}(B) \rightarrow 1
$$

and $\pi_{1}(F)$ acts nilpotently on $\pi_{i}(F)$. Thus $Y_{i+1} \rightarrow Y_{i}$ is a fibration of the type discussed in Lemma 3.3.

The converse of Theorem 3.4 is also true, for the restriction to the fiber of factorizations as in the theorem are principal refinements of the Postnikov tower of $F$. 
4. The case of a nilpotent fiber. In this section we shall prove the main result in its full generality.

In fact, all preparations have been made already so we proceed straightway to the formulation of

THEOREM 4.1. Suppose that the fiber $F$ of $p: Y \rightarrow B$ is nilpotent and that either $(X, A)$ is finitely coconnected or $F$ is finitely anticonnected. Then $F_{u}(X, A ; Y, B)$ is nilpotent.

In order to prove this statement, replace the "crude" Postnikov tower by the refined one of Theorem 3.4 and proceed as in Proposition 2.5.

For an application of Theorem 4.1, suppose that $F$ is a $G$-space and $\bar{X}$ a free (principal) $G$-space such that $(\bar{X} / G, \bar{A} / G)=(X, A)$ for some $G$-invariant subspace $\bar{A} \subset \bar{X}$. Denote by $F_{f}(\bar{X}, \bar{A} ; F)^{G}$ the space of all $G$-equivariant extensions of $f \mid \bar{A}$, where $f: \bar{X} \rightarrow F$ is some $G$-map.

Corollary 4.2. $F_{f}(\bar{X}, \overline{A ;} F)^{G}$ is nilpotent under the assumptions of Theorem 4.1.

Proof. Apply the theorem to the space of sections of the associated fiber bundle $\bar{X} \times{ }_{G} F \rightarrow X$.

In particular, section spaces and equivariant function spaces admit localizations. That these localizations behave as expected will be shown in the next, final section.

5. Localizations of section spaces. Throughout this section we make the additional assumption that $(X, A)$ is a relative $C W$-complex with finite skeleta.

For any family $P$ of primes, the $P$-localized module $M_{P}$ is canonically a $G$-module and the localization map $e: M \rightarrow M_{P}$ a $G$-homomorphism.

Given a homomorphism $\pi_{1}(X) \rightarrow G, M$ and $M_{P}$ become local coefficient systems in $X$.

LEMMA 5.1. The coefficient group homomorphism

$$
e_{*}: H^{*}(X, A ; M) \rightarrow H^{*}\left(X, A ; M_{P}\right)
$$

is a P-localization.

Proof. Let $\Gamma^{*}(X, A ; M), \Gamma^{*}\left(X, A ; M_{P}\right)$ denote the cellular cochain complexes of ( $X, A)$ with local coefficients $M, M_{P}$. Since localization commutes with direct sum, the commutative diagram

$$
\begin{array}{cccc}
\bigoplus_{\alpha} M\left(h_{\alpha}\left(E_{0}\right)\right) & \stackrel{\cong}{ } & \Gamma^{q}(X, A ; M) \\
\downarrow e & & \downarrow e_{*} \\
\bigoplus_{\alpha} M\left(h_{\alpha}\left(E_{0}\right)\right)_{P} & \stackrel{\cong}{\rightarrow} & \Gamma^{q}\left(X, A ; M_{P}\right)
\end{array}
$$

where $\left\{h_{\alpha}: \Delta^{q} \rightarrow X\right\}$ are the $q$-cells of $(X, A)$ and $E_{0} \in \Delta^{q}$ the base point, shows that $e_{*} P$-localizes on the cochain level. To complete the proof, observe that $e_{*}$ is a cochain map and localization an exact functor. 
Let also $e: L \rightarrow L_{(P)}$ denote the $\mathscr{T} K(G, 1)$-morphism of $L:=L(M, n+1)$ into $L_{(P)}:=L\left(M_{P}, n+1\right), n>0$, corresponding to $e: M \rightarrow M_{P}$.

In the following,

$$
F_{u}^{0}(-,-;-,-)
$$

denotes the path-component of $F_{u}(-,-;-,-)$ containing $u ; \underline{m}$, where $m$ is a fiber map, denotes the map on function spaces defined as post composition with $m$.

Lemma 5.2. For any map $v: X \rightarrow \bar{P} L$,

$$
\underline{\bar{P} e}: F_{v}^{0}(X, A ; \bar{P} L, L) \rightarrow F_{P_{e l}}^{0}\left(X, A ; \bar{P} L_{(P)} ; L_{(P)}\right)
$$

is a P-localization.

Proof. This follows from Lemma 5.1 and the decomposition [12] of the function spaces in question as products of Eilenberg-Mac Lane spaces.

Consider the fiberwise $P$-localization $e: Y \rightarrow Y_{(P)}$ constructed in $[1,9]$ for any fibration with nilpotent fiber.

THEOREM 5.3. Suppose that $F$ is nilpotent and that either $(X, A)$ is finite or $F$ is finitely anticonnected. Then

$$
\text { e: } F_{u}^{0}(X, A ; Y, B) \rightarrow F_{e u}^{0}\left(X, A ; Y_{(P)}, B\right)
$$

is a P-localization.

The proof is again by induction in the refined Postnikov tower of $p$ and proceeds as that of [6, Theorem B] once [6, Theorem 2.1] has been replaced by Lemma 5.2.

In the situation of Corollary 4.2 and under the same assumptions as in Theorem 5.3 we have

Corollary 5.4. $F_{f}^{0}(\bar{X}, \bar{A} ; F)_{P}^{G}=F_{e f}^{0}\left(\bar{X}, \bar{A} ; F_{P}\right)^{G}$.

Proof. $\left(\bar{X} \times{ }_{G} F\right)_{(P)}=\bar{X} \times{ }_{G} F_{P}$.

We finish this paper by considering an application of Corollary 5.4.

EXAmple 5.5. The antipodal map defines a $\mathbf{Z} / 2$-action on $S^{n}$. Suppose that $n$ is even (and positive) and let 1 denote the identity map. Then any component of $F_{1}\left(S^{n}, S^{m} ; S^{n}\right)^{\mathbf{Z} / 2}$ is rationally homotopy equivalent to $S^{n-m-1}$ if $m$ is even, $0 \leqslant m<n$, and to $S^{2 n-m-2}$ if $m$ is odd, $-1 \leqslant m<n$ (where $S^{-1}=\varnothing$ ).

The assertions in Example 5.5 follow from the decomposition [12] of the section space for $S^{n} \times_{\mathbf{z} / 2} S_{(0)}^{n} \rightarrow \mathbf{R} P^{n}$ induced from the Postnikov tower; cf. [3, 13]. Note that the results of [ $\mathbf{3}$ or $\mathbf{1 5}$ ] cannot be used as neither the fibration nor the base is nilpotent.

Added in proof. Only $K\left(\pi_{1}(B), 1\right)$-principal fibrations are needed in Theorem 3.4 since in Lemma 3.1. Out $(N)$ can easily be replaced by $\pi_{1}(B)$.

\section{REFERENCES}

1. A. K. Bousfield and D. M. Kan, Homotopy limits, completions and localizations, Lecture Notes in Math.. vol. 304. Springer-Verlag, Berlin-Heidelberg-New York, 1972.

2. H. Federer, A stud of function spaces by spectral sequences, Trans. Amer. Math. Soc. 82 (1956), $340-361$.

3. A. Hacfliger, Rational homotopy of the space of sections of a nilpotent bundle. Trans. Amer. Math. Soc. 273 (1982), 609-620. 
4. R. O. Hill, Jr., Moore-Postnikov towers for fibrations in which $\pi_{1}$ (fibre) is non-abelian, Pacific J. Math. 62 (1976), 141-148.

5. P. Hilton, G. Mislin and J. Roitberg, Localization of nilpotent groups and spaces, North-Holland Mathematics Studies no. 15, North-Holland, Amsterdam, 1975.

6. P. Hilton, G. Mislin, J. Roitberg and R. Steiner, On free maps and free homotopies into nilpotent spaces, Algebraic Topology (Proceedings, Vancouver 1977), Lecture Notes in Math., vol. 673, SpringerVerlag, Berlin-Heidelberg-New York, 1978.

7. D. Husemoller, Fibre bundles, Graduate Texts in Math., vol. 20, Springer-Verlag, Berlin-HeidelbergNew York, 1975.

8. L. L. Larmore and E. Thomas, On the fundamental group of a space of sections, Math. Scand. 47 (1980), 232-246.

9. I. Llerena, Localization of fibrations with nilpotent fibre, Math. Z. 188 (1985), 397-410.

10. J. F. McClendon, Obstruction theory in fiber spaces, Math. Z. 120 (1971), 1-17.

11. Higher order twisted cohomology operations, Invent. Math. 7 (1969), 183-214.

12. J. M. Møller, Spaces of sections of Eilenberg-Mac Lane fibrations, Pacific J. Math. 129 (1987).

13. J. M. Møller and M. Raussen, Rational homotopy of spaces of maps into spheres and complex projective spaces, Trans. Amer. Math. Soc. 292 (1985), 721-732.

14. C. A. Robinson, Moore-Postnikov systems for non-simple fibrations, Illinois J. Math. 16 (1972), 234-242.

15. F. E. A. da Silveira, Rational homotopy theory of fibrations, Pacific J. Math. 113 (1984), 1-34.

Matematisk Institut, Universitetsparken 5, DK - 2100 Copenhagen $\varnothing$, Denmark 